\title{
Study The Effect of Factors on the Rate Constant (K) for Some Substituted Benzyl-amine Using Theoretical Calculations
}

\author{
Ammar A. Ibrahim ${ }^{1 *}$, Huda A. Younis ${ }^{2}$, Entesar A. Sulliman ${ }^{3}$, Maher A. Ibrahim ${ }^{4}$ Zahraa O. Yaareb ${ }^{1}$ \\ ${ }^{1}$ Depart. of Chemistry, College of Science, University of Mosul, Iraq \\ ${ }^{2}$ Northern Technical University, Technical Institute, Mosul, Iraq \\ ${ }^{3}$ Al-Noor University College, Iraq \\ ${ }^{4}$ Department of Biochemistry, College of Medicine, University of Mosul, Iraq \\ *ammar74@uomosul.edu.iq
}

\begin{abstract}
The computational calculations methods are applied for the determination of some benzylamine substituents. The $\log \mathrm{K}$ at temperature $303 \mathrm{~K}$ are used as dependent variables. While the other variables like HOMO, LUMO, Dipole moment, chemical potential, hardness, softness and electrophilicity are independent variables. AM1, PM3 and HF/STO-3G methods have been utilized to portend $(\log \mathrm{K})$ parameters. The fisher values is taken as a criterion for our system to predict the $(\operatorname{logK})$ parameters.
\end{abstract}

Keywords: Rate Constant, Benzyl-amine, HOMO, LUMO

\section{Introduction:}

Many compounds were synthesized by treated with benzyl-amine[1-3] or coupling with benzylamine[4]. The reactivity of some compounds in the presence of different amine like benzyl-amine have been determinate. And the mechanism of de-oxygenated for the product was exanimate[5]. Complexes of benzylamine as ligand have been synthesis by researchers[6,7]. The instrumentals ${ }^{1} \mathrm{HNMR}$, IR and UV-Vis spectroscopy have been applied to identify the ligand complexes[8,9]. Benzyl-amine used as a reagent to prepare compounds like Pyrrolidino. The voltammetric instrumental (cyclic voltammetry) was been used to measure and characterized the reactions[10].

Also, the transfer of proton in the substituted of benzyl-amine was proposed in the protic solvents[11]. The equipment of HPLC was utilized the basicity of benzyl-amine which generated from ortho-substituted of aromatic groups[12]. Te theoretical calculations were useful and applied in many kinds of research to evaluate the relationship between the physic-chemical parameters[13-16]. The Structure-activity relationships (SAR) and quantitative structure-activity relationships (QSAR) were used for the benzyl-amine derivatives to evaluate the correlation between the electronic effect[17,18].

Imines or Shiff bases complexes were produced by the oxidation coupling of benzyl-amine using the Co-catalyzed[19] or self-coupling[20]. B3PW91/6-31+G(d) method was used to calculate the physical parameters of the (2-benzyl-amino-1,4-naphthalene-dione) compound. Inter and intramolecular hydrogen bonding have been characterized[21]. Benzyl-amine was used to synthesize of compounds[22,23] which applied clinically to development of the anti-bacterial drugs[24-26]. Zeroorder kinetic was noticed in the hydrogenolysis of benzyl-amine which lead to adsorption of this compound at the surface[27].

Semi-empirical determination MNDO-PM3 was used to predict the different amines like benzyl-amine depending on the charges of some atoms[28]. Spectroscopic and theoretical calculations methods have been applied to the determination of the rate constant for the addition of benzyl-amine depends on the Michael addition[29].

\section{Computational Methods:}

The theoretical of the calculations methods AM1, PM3 and HF/STO-3G have been carried out by Chembio office 13 package. First, the model was minimized by MM2 to reach the best configuration depending on the less steric energy and more stable. Later, the model was determined by the other methods AM1, PM3 and HF/STO-3G. So, the physical properties of the substituted are calculated and tabulated to compare with the others. 
SPSS program was applied as a statistical method to compute and calculate the parameters. Single and multiple linear regression were used to determine the correlation between the practical values as dependent with calculated parameters as an independent.

\section{Results and Discussion:}

Figure (1) represents the structure of the derivative of benzyl-amine compounds. While table (1) listed the practical results of $\log \mathrm{K}$ and Hammett standard values for benzyl-amine groups[30].

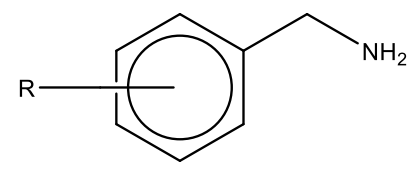

Benzylamine

Figure 1. Benzyl-amine structure

Table1. Segma and rate constant values for derivatives benzyl-amine at temperature $303 \mathrm{~K}$

\begin{tabular}{lll}
\hline Substituent & Segma & $\begin{array}{l}\text { Log } \\
303 \mathrm{~K}\end{array}$ \\
\hline $\mathrm{H}$ & 0 & 1.279 \\
$\mathrm{~m}-\mathrm{CF} 3$ & 0.43 & 0.262 \\
$\mathrm{~m}-\mathrm{CH} 3$ & -0.07 & 1.412 \\
$\mathrm{~m}-\mathrm{F}$ & 0.34 & 0.610 \\
$\mathrm{~m}-\mathrm{I}$ & 0.35 & 0.679 \\
$\mathrm{~m}-\mathrm{NH} 2$ & -0.16 & 1.599 \\
$\mathrm{~m}-\mathrm{OCH} 3$ & 0.12 & 1.217 \\
$\mathrm{p}-\mathrm{Br}$ & 0.23 & 1.000 \\
$\mathrm{p}-\mathrm{CF} 3$ & 0.54 & 0.107 \\
$\mathrm{p}-\mathrm{Cl}$ & 0.23 & 1.124 \\
\hline
\end{tabular}

Tables (2-4) were presented the physical properties of the substituted of the benzyl-amine which the AM1, PM3 and HF/STO-3G have been applied.

Table 2. Physico-chemical parameters for benzyl-amine substituted using AM1 method

\begin{tabular}{|c|c|c|c|c|c|c|c|c|c|}
\hline $\begin{array}{l}\text { Benzyl } \\
\text { amine }\end{array}$ & $\begin{array}{l}\log \\
303 \mathrm{~K}\end{array}$ & H.F & HOMO & Dipole & LUMO & $\begin{array}{l}\text { Chemical } \\
\text { potential }\end{array}$ & $\begin{array}{l}\text { Hard- } \\
\text { ness }\end{array}$ & $\begin{array}{l}\text { Soft- } \\
\text { ness }\end{array}$ & $\begin{array}{l}\text { Electro- } \\
\text { philicity }\end{array}$ \\
\hline $\mathrm{H}$ & 1.279 & 19.68 & -9.351 & 1.466 & 0.414 & -4.469 & 4.883 & -3.883 & 2.045 \\
\hline $\mathrm{m}-\mathrm{CF} 3$ & 0.262 & -137.29 & -9.985 & 2.798 & -0.269 & -5.127 & 4.858 & -3.858 & 2.705 \\
\hline $\mathrm{m}-\mathrm{CH} 3$ & 1.412 & 11.69 & -9.291 & 1.103 & 0.459 & -4.416 & 4.875 & -3.875 & 2.000 \\
\hline $\mathrm{m}-\mathrm{F}$ & 0.610 & -25.54 & -9.489 & 1.442 & 0.102 & -4.693 & 4.795 & -3.795 & 2.297 \\
\hline m-I & 0.679 & 35.81 & -9.559 & 1.289 & 0.033 & -4.763 & 4.796 & -3.796 & 2.365 \\
\hline m-NH2 & 1.599 & 18.25 & -8.564 & 1.700 & 0.500 & -4.032 & 4.532 & -3.532 & 1.794 \\
\hline $\mathrm{m}-\mathrm{OCH} 3$ & 1.217 & -20.87 & -9.106 & 2.620 & 0.284 & -4.411 & 4.695 & -3.695 & 2.072 \\
\hline $\mathrm{p}-\mathrm{Br}$ & 1.000 & 24.46 & -9.416 & 2.141 & -0.039 & -4.727 & 4.688 & -3.688 & 2.383 \\
\hline $\mathrm{p}-\mathrm{CF} 3$ & 0.107 & -137.17 & -10.035 & 4.080 & -0.352 & -5.194 & 4.842 & -3.842 & 2.786 \\
\hline $\mathrm{p}-\mathrm{Cl}$ & 1.124 & 12.50 & -9.370 & 2.007 & 0.049 & -4.661 & 4.710 & -3.710 & 2.306 \\
\hline
\end{tabular}


Table 3. Physico-chemical parameters for the benzyl-amine substituted using PM3 method

\begin{tabular}{|c|c|c|c|c|c|c|c|c|c|}
\hline $\begin{array}{l}\text { Benzyl } \\
\text { amine }\end{array}$ & $\begin{array}{l}\log \\
303 \mathrm{~K} \\
\end{array}$ & H.F & HOMO & Dipole & LUMO & $\begin{array}{l}\text { Chemical } \\
\text { potential }\end{array}$ & $\begin{array}{l}\text { Hard- } \\
\text { ness }\end{array}$ & $\begin{array}{l}\text { Soft- } \\
\text { ness }\end{array}$ & $\begin{array}{l}\text { Electro- } \\
\text { philicity }\end{array}$ \\
\hline $\mathrm{H}$ & 1.279 & 21.84 & -9.361 & 1.253 & 0.296 & -4.532 & 4.828 & -3.828 & 2.127 \\
\hline $\mathrm{m}-\mathrm{CF} 3$ & 0.262 & -136.69 & -9.700 & 2.654 & -0.454 & -5.077 & 4.623 & -3.623 & 2.788 \\
\hline $\mathrm{m}-\mathrm{CH} 3$ & 1.412 & 12.51 & -9.310 & 1.024 & 0.306 & -4.502 & 4.808 & -3.808 & 2.108 \\
\hline $\mathrm{m}-\mathrm{F}$ & 0.610 & -21.80 & -9.540 & 1.316 & -0.028 & -4.784 & 4.756 & -3.756 & 2.406 \\
\hline $\mathrm{m}-\mathrm{I}$ & 0.679 & 43.13 & -9.041 & 0.964 & -0.442 & -4.742 & 4.300 & -3.300 & 2.614 \\
\hline m-NH2 & 1.599 & 19.80 & -8.616 & 1.533 & 0.334 & -4.141 & 4.475 & -3.475 & 1.916 \\
\hline m-OCH3 & 1.217 & -16.07 & -9.125 & 1.213 & 0.242 & -4.441 & 4.683 & -3.683 & 2.106 \\
\hline $\mathrm{p}-\mathrm{Br}$ & 1.000 & 29.46 & -9.517 & 1.972 & -0.040 & -4.778 & 4.738 & -3.738 & 2.409 \\
\hline $\mathrm{p}-\mathrm{CF} 3$ & 0.107 & -136.63 & -9.731 & 3.868 & -0.526 & -5.128 & 4.602 & -3.602 & 2.857 \\
\hline $\mathrm{p}-\mathrm{Cl}$ & 1.124 & 15.05 & -9.284 & 1.772 & -0.013 & -4.648 & 4.635 & -3.635 & 2.331 \\
\hline
\end{tabular}

Table 4. Physico-chemical parameters for the benzyl-amine substituted using HF/STO-3G method

\begin{tabular}{llllllllll}
\hline $\begin{array}{l}\text { Benzyl } \\
\text { amine }\end{array}$ & log & H.F & HOMO & Dipole & LUMO & $\begin{array}{l}\text { Chemical } \\
\text { potential }\end{array}$ & $\begin{array}{l}\text { Hard- } \\
\text { ness }\end{array}$ & $\begin{array}{l}\text { Soft- } \\
\text { ness }\end{array}$ & $\begin{array}{l}\text { Electro- } \\
\text { philicity }\end{array}$ \\
\hline $\mathrm{H}$ & 1.279 & -320.78 & -0.271 & 1.496 & 0.270 & 0.000 & 0.271 & 0.729 & $7.39 \mathrm{e}-8$ \\
$\mathrm{~m}-\mathrm{CF} 3$ & 0.262 & -651.75 & -0.287 & 1.944 & 0.245 & -0.021 & 0.266 & 0.734 & $8.47 \mathrm{e}-4$ \\
$\mathrm{~m}-\mathrm{CH} 3$ & 1.412 & -359.37 & -0.265 & 1.247 & 0.272 & 0.003 & 0.268 & 0.732 & $2.10 \mathrm{e}-5$ \\
$\mathrm{~m}-\mathrm{F}$ & 0.610 & -418.24 & -0.263 & 1.429 & 0.261 & -0.001 & 0.262 & 0.738 & $1.69 \mathrm{e}-6$ \\
$\mathrm{~m}-\mathrm{I}$ & 0.679 & -7170.90 & -0.248 & 1.764 & 0.255 & 0.003 & 0.252 & 0.748 & $2.28 \mathrm{e}-5$ \\
$\mathrm{~m}-\mathrm{NH} 2$ & 1.599 & -375.11 & -0.237 & 2.417 & 0.271 & 0.017 & 0.254 & 0.746 & $6.01-4$ \\
$\mathrm{~m}-\mathrm{OCH} 3$ & 1.217 & -433.20 & -0.243 & 0.801 & 0.269 & 0.013 & 0.256 & 0.744 & $3.32 \mathrm{e}-4$ \\
p-Br & 1.000 & -2864.88 & -0.256 & 2.733 & 0.254 & -0.001 & 0.255 & 0.745 & $3.21 \mathrm{e}-6$ \\
p-CF3 & 0.107 & -651.75 & -0.288 & 3.118 & 0.242 & -0.023 & 0.265 & 0.735 & $9.97 \mathrm{e}-4$ \\
p-Cl & 1.124 & -774.79 & -0.280 & 3.748 & 0.244 & -0.018 & 0.262 & 0.738 & $6.32 \mathrm{e}-4$ \\
\hline
\end{tabular}

Tables (5-7) were displayed the comparison between calculated physico-chemical parameters with experimental values in different methods of calculations.

Table 5. Bivariate correlation for the benzyl-amine substituted using AM1 method

\begin{tabular}{llllllllll}
\hline AM1 & $\begin{array}{l}\text { Log } \\
303 \mathrm{~K}\end{array}$ & Sigma & Dipole & HOMO & LUMO & $\begin{array}{l}\text { Chem. } \\
\text { Potential }\end{array}$ & $\begin{array}{l}\text { Hard- } \\
\text { ness }\end{array}$ & $\begin{array}{l}\text { Soft- } \\
\text { ness }\end{array}$ & $\begin{array}{l}\text { Electro- } \\
\text { philicity }\end{array}$ \\
\hline $\log 303 \mathrm{~K}$ & 1 & & & & & & & & \\
Sigma & -0.961 & 1 & & & & & & & \\
Dipole & -0.625 & 0.608 & 1 & & & & & & \\
HOMO & 0.909 & -0.879 & -0.532 & 1 & & & & & \\
LUMO & 0.924 & -0.952 & -0.716 & 0.863 & 1 & & & & \\
Chem. & 0.947 & -0.941 & -0.630 & 0.976 & 0.952 & 1 & & & \\
Potential & -0.486 & 0.392 & 0.053 & -0.739 & -0.297 & -0.576 & 1 & & \\
Hardness & -0.963 & & & & \\
Softness & 0.486 & -0.392 & -0.053 & 0.739 & 0.297 & 0.576 & -1.000 & 1 & \\
$\begin{array}{l}\text { Electro- } \\
\text { philicity }\end{array}$ & -0.949 & 0.953 & 0.687 & -0.937 & -0.985 & -0.990 & 0.457 & -0.457 & 1 \\
\hline
\end{tabular}


Table 6. Bivariate correlation for the benzyl-amine substituted using AM1 method

\begin{tabular}{llllllllll}
\hline PM3 & $\begin{array}{l}\text { Log } \\
\text { 303K }\end{array}$ & Sigma & Dipole & HOMO & LUMO & $\begin{array}{l}\text { Chem. } \\
\text { Potential }\end{array}$ & $\begin{array}{l}\text { Hard- } \\
\text { ness }\end{array}$ & $\begin{array}{l}\text { Soft- } \\
\text { ness }\end{array}$ & $\begin{array}{l}\text { Electro- } \\
\text { philicity }\end{array}$ \\
\hline log303K & 1 & & & & & & & & \\
Sigma & -0.961 & 1 & & & & & & & \\
Dipole & -0.701 & 0.641 & 1 & & & & & & \\
HOMO & 0.736 & -0.708 & -0.598 & 1 & & & & & \\
LUMO & 0.927 & -0.927 & -0.640 & 0.542 & 1 & & & & \\
Chem. & 0.947 & -0.931 & -0.705 & 0.877 & 0.879 & 1 & & & \\
Potential & & & & & & & & \\
Hardness & 0.202 & -0.231 & -0.046 & -0.476 & 0.481 & 0.005 & 1 & & \\
Softness & -0.202 & 0.231 & 0.046 & 0.476 & -0.481 & -0.005 & -1.000 & 1 & \\
$\begin{array}{l}\text { Electro- } \\
\text { philicity }\end{array}$ & -0.966 & 0.954 & 0.697 & -0.711 & -0.976 & -0.961 & -0.279 & 0.279 & 1 \\
\hline
\end{tabular}

Table 7. Bivariate correlation for the benzyl-amine substituted using AM1 method

\begin{tabular}{llllllllll}
\hline HF/STO & $\begin{array}{l}\text { Log } \\
\text { 303K }\end{array}$ & Sigma & Dipole & HOMO & LUMO & $\begin{array}{l}\text { Chem. } \\
\text { Potential }\end{array}$ & $\begin{array}{l}\text { Hard- } \\
\text { ness }\end{array}$ & $\begin{array}{l}\text { Soft- } \\
\text { ness }\end{array}$ & $\begin{array}{l}\text { Electro- } \\
\text { philicity }\end{array}$ \\
\hline $\log 303 \mathrm{~K}$ & 1 & & & & & & & & \\
Sigma & -0.961 & 1 & & & & & & & \\
Dipole & -0.208 & 0.307 & 1 & & & & & & \\
HOMO & 0.616 & -0.571 & -0.416 & 1 & & & & & \\
LUMO & 0.777 & -0.852 & -0.709 & 0.680 & 1 & & & & \\
Chem. & 0.742 & -0.746 & -0.578 & 0.946 & 0.881 & 1 & & & \\
$\begin{array}{l}\text { Potential } \\
\text { Hardness }\end{array}$ & -0.143 & 0.013 & -0.078 & -0.749 & -0.025 & -0.493 & 1 & & \\
$\begin{array}{l}\text { Softness } \\
\text { Electro- } \\
\text { philicity }\end{array}$ & 0.143 & -0.013 & 0.078 & 0.749 & 0.025 & 0.493 & -1.000 & 1 & \\
\hline
\end{tabular}

The employed of the equations to calculate the $\log \mathrm{K}$ theoretically depends on the calculated physical properties. The multiple linear regression method as the statistical method was used to evaluate and predicted the practical values. The equations showed different methods of the calculations AM1, PM3 and HF/STO-3G. For each theoretical method, two statistical methods (enter and stepwise) were applied to predicted the values.

AM1:

Enter Method:

$\log \mathrm{K}=2.277-2.600($ segma $)+0.086($ dipole $)-18.879($ LUMO) +9.366 (Hardness $)-19.087$ (Electrophilicity)

$\mathrm{R}=0.993$, St.error $=0.084, \mathrm{~F}=60.64$

Stepwise Method:

$\log \mathrm{K}=1.352-2.107$ (segma)

$\mathrm{R}=0.961$, St. error $=0.145, \mathrm{~F}=97.13$

PM3:

Enter Method:

$\log \mathrm{K}=3.949-1.742($ segma $)+0.015$ (dipole) -12.367 (Chempotential) -6.643 (Hardness $)-12.546$ (Electrophilicity)

$\mathrm{R}=0.987$, St. error $=0.121, \mathrm{~F}=29.30$

Stepwise Method:

$\log \mathrm{K}=4.558-1.534$ (electrophilicity)

$\mathrm{R}=0.966$, St. error $=0.135, \mathrm{~F}=111.90$ 
Enter Method:

$\log \mathrm{K}=-101.535-2.602($ segma $)-0.018($ dipole $)-124.587($ HOMO $)-42.353($ LUMO $)+150.817$

(Chemical potential) +110.445 (Softness) -251.18 (Electrophilicity)

$\mathrm{R}=0.984$, St. error $=0.188, \mathrm{~F}=8.617$

Stepwise Method:

$\log \mathrm{K}=1.352-2.107$ (segma)

$\mathrm{R}=0.961$, St. error $=0.145, \mathrm{~F}=97.13$

Table (8) appeared the predicted values of the $\log \mathrm{K}$ which calculated in different theoretical methods. Also, the correlation coefficient (R), the standard error and the fisher values have been determined.

Table 8. The predicted of $\log \mathrm{K}$ for the benzyl-amine substituted using different methods of calculations

\begin{tabular}{llllllll}
\hline & & AM1 & & PM3 & \multicolumn{3}{c}{ HF/STO-3G } \\
& Practical & Enter & stepwise & Enter & stepwise & Enter & stepwise \\
\hline $\mathrm{H}$ & 1.279 & 1.288 & 1.352 & 1.256 & 1.295 & 1.252 & 1.352 \\
$\mathrm{~m}-\mathrm{CF} 3$ & 0.262 & 0.339 & 0.446 & 0.341 & 0.281 & 0.386 & 0.446 \\
$\mathrm{~m}-\mathrm{CH} 3$ & 1.412 & 1.372 & 1.499 & 1.379 & 1.325 & 1.446 & 1.499 \\
$\mathrm{~m}-\mathrm{F}$ & 0.610 & 0.666 & 0.636 & 0.759 & 0.867 & 0.626 & 0.636 \\
$\mathrm{~m}-\mathrm{I}$ & 0.679 & 0.631 & 0.615 & 0.629 & 0.547 & 0.803 & 0.615 \\
$\mathrm{~m}-\mathrm{NH} 2$ & 1.599 & 1.612 & 1.689 & 1.697 & 1.619 & 1.684 & 1.689 \\
$\mathrm{~m}-\mathrm{OCH} 3$ & 1.217 & 1.252 & 1.099 & 1.152 & 1.327 & 1.063 & 1.099 \\
$\mathrm{p}-\mathrm{Br}$ & 1.000 & 1.020 & 0.867 & 0.967 & 0.862 & 1.088 & 0.867 \\
$\mathrm{p}-\mathrm{CF} 3$ & 0.107 & 0.047 & 0.214 & 0.068 & 0.175 & 0.098 & 0.214 \\
$\mathrm{p}-\mathrm{Cl}$ & 1.124 & 1.021 & 0.867 & 1.028 & 0.983 & 0.989 & 0.867 \\
\hline $\mathrm{R}$ & & 0.993 & 0.961 & 0.987 & 0.966 & 0.980 & 0.961 \\
St. error & & 0.060 & 0.145 & 0.085 & 0.135 & 0.104 & 0.145 \\
Fisher value & & 605.85 & 97.04 & 296.59 & 112.00 & 194.62 & 97.04 \\
\hline
\end{tabular}

While table (9) was showed the percent error of the parameters compare with the practical values. This (\%error) values were calculated depends on the following equation :

$$
\text { Percent }=\frac{\text { theoretical }- \text { experimental }}{\text { theoretical }} \times 100
$$

Table(9): Percent error for predicted benzyl-amine derivatives

\begin{tabular}{lllllll}
\hline & \multicolumn{2}{l}{ AM1 (\%error) } & \multicolumn{2}{l}{ PM3 (\%error) } & \multicolumn{2}{l}{ HF/STO-3G (\%error) } \\
& Enter & stepwise & Enter & stepwise & Enter & stepwise \\
\hline $\mathrm{H}$ & 0.68 & 5.42 & -1.79 & 1.24 & -2.13 & 5.42 \\
$\mathrm{~m}-\mathrm{CF} 3$ & 22.64 & 41.15 & 22.97 & 6.74 & 31.92 & 41.15 \\
$\mathrm{~m}-\mathrm{CH} 3$ & -2.92 & 5.86 & -2.34 & -6.56 & 2.36 & 5.86 \\
$\mathrm{~m}-\mathrm{F}$ & 8.52 & 4.09 & 19.74 & 29.71 & 2.64 & 4.09 \\
$\mathrm{~m}-\mathrm{I}$ & -7.62 & -10.56 & -7.99 & -24.13 & 15.37 & -10.56 \\
$\mathrm{~m}-\mathrm{NH} 2$ & 0.84 & 5.35 & 5.80 & 1.24 & 5.07 & 5.35 \\
$\mathrm{~m}-\mathrm{OCH} 3$ & 2.77 & -10.76 & -5.70 & 8.28 & -14.51 & -10.76 \\
p-Br & 1.94 & -15.29 & -3.39 & -16.01 & 8.05 & -15.29 \\
p-CF3 & -127.85 & 49.95 & -56.88 & 38.71 & -9.50 & 49.95 \\
p-Cl & -10.08 & -29.57 & -9.37 & -14.37 & -13.67 & -29.57 \\
\hline Average & -11.11 & 4.56 & -3.90 & 2.49 & 2.56 & 4.56 \\
\hline
\end{tabular}

Figures (2) showed the fisher values of the benzyl-amine derivatives which determination at different methods of the calculations and different statistical methods. While figure (3) was presented the average of the percent error (\%error) for all methods and at different statistical methods. 


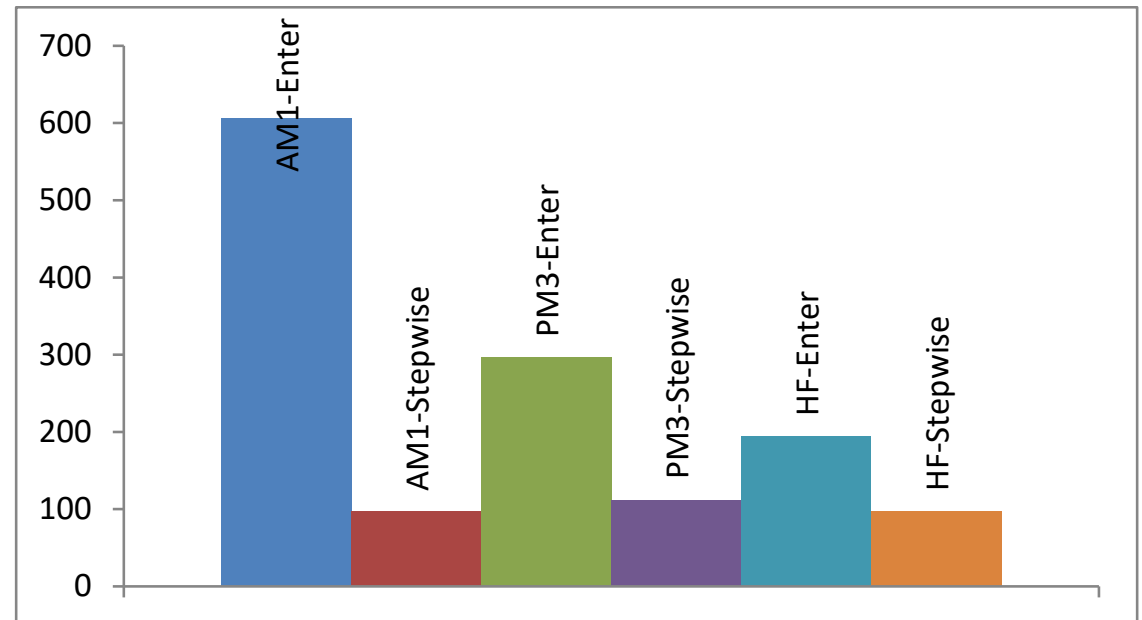

Fig 2. Fisher values calculated at different methods

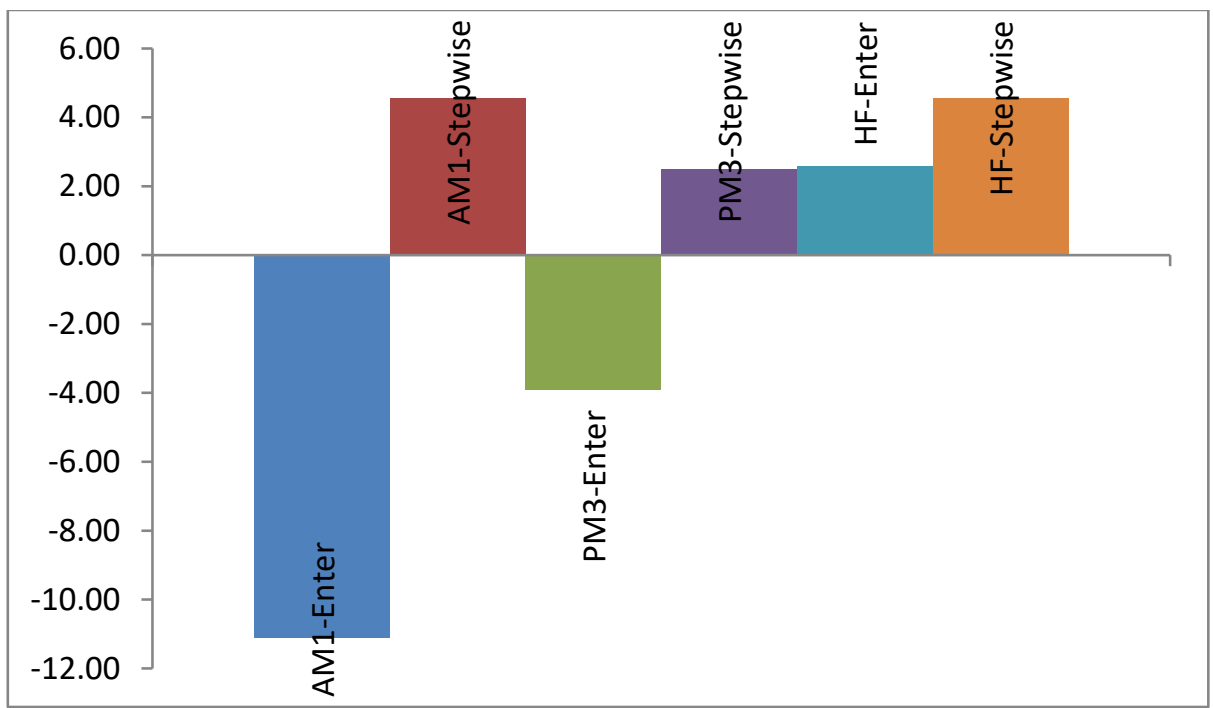

Fig 3. Average parameters at various methods of calculation

\section{Conclusions:}

Different variations of the substituted of benzyl-amine compounds were determined theoretically using different methods. The correlation between the predicted values and the practical showed excellent correlations. The equations for each method AM1, PM3 and HF/STO-3G were given a model to predicted the $(\log K)$ data. Finally, the fisher values or the (\%error) data were an indicated parameter to calculated or predicted the practical data depends on the model or on the used methods.

\section{References:}

[1] Akihito Hirabayashi, Harunobu Mukaiyama, Hiroaki Kobayashi, Hiroaki Shiohara, Satoko Nakayama, Motoyasu Ozawa, Keiji Miyazawa, Keiko Misawa, Hideki Ohnota, Masayuki Isaji, Bioorganic \& Medicinal Chemistry 17 (2009) 284-294.

[2] Heather A. Barnett, Diane M. Coe, Tony W. J. Cooper, Torquil I. Jack, Haydn T. Jones, Simon J. F. Macdonald, Iain M. McLay, Natalie Rayner, Rosemary Z. Sasse, Tracy J. Shipley, Phil A. Skone, Graham I. Somers, Simon Taylor, Iain J. Uings, James M. Woolven, Gordon G. Weingarten, Bioorganic \& Medicinal Chemistry Letters 19 (2009) 158-162

[3] C. Baggiani, L. Anfossi, P. Baravalle, C. Giovannoli, C. Tozzi, Analytica Chimica Acta 531 (2005) 199-207.

[4] Yasutomi Asano, Shuji Kitamura, Taiichi Ohra, Fumio Itoh, Masahiro Kajino, Tomoko Tamura, Manami Kaneko, Shota Ikeda, Hideki Igata, Tomohiro Kawamoto, Satoshi Sogabe, Shin-ichi 
Matsumoto, Toshimasa Tanaka, Masashi Yamaguchi, Hiroyuki Kimurab and Shoji Fukumoto, Bioorganic \& Medicinal Chemistry 16 (2008) 4699-4714.

[5] Hiroyuki Morita, Shintaro Tashiro, Masahiro Takeda, Nobuhiko Yamada, Md. Chanmiya Sheikh, Hiroyuki Kawaguchi, Tetrahedron 64 (2008) 4496e4505

[6] Joan Albert, J. Magali Cadena, Jaume Granell, Xavier Solans, Merce` Font-Bardia, Journal of Organometallic Chemistry 689 (2004) 4889-4896

[7] Veli T. Kasumov, Ahmet Bulut, Fevzi Ko“ksal, Mehmet Aslanog`lu,_Ibrahim Uc,ar, Canan Kazak, Polyhedron 25 (2006) 1133-1141

[8] Raha, K. et al. (2005) Pairwise decomposition of residue interaction energies using semiempirical quantum mechanical methods in studies of protein-ligand interaction. J. Am. Chem. Soc. 127, 6583-6594

[9] Soraia Meghdadi, Mehdi Amirnasr, Mohammad H. Habibi, Ahmad Amiri, Vahid Ghodsi, Azadeh Rohani, Ross W. Harrington and William Clegg, Polyhedron 27 (2008) 2771-2778

[10] Pavel A. Troshin, Alexey B. Kornev, Alexander S. Peregudov, Svetlana M. Peregudova and Rimma N. Lyubovskaya, Mendeleev Commun., 2007, 17, 116-118.

[11] D. Guha, A. Mandal, A. Koll, A. Filarowski, S. Mukherjee, Spectrochim. Acta A 56 (2000) 2669.

[12] M. Bartolini, C. Bertucci, R. Gotti, V. Tumiatti, A. Cavalli, M. Recanatini, V. Andrisano, Journal of Chromatography A, 958 (2002) 59-67

[13] Ammar A. Ibrahim, Ghufran Mohammed Abed, International Journal of Scientific \& Engineering Research Volume 9, Issue 1, January-2018.

[14] Ammar A. Ibrahim, Eid A. Abdalrazaq, American Journal of Applied Sciences 6 (7): 1385-1389, 2009.

[15] Ammar A. Ibrahim, Eid A. Abdalrazaq, Maher A. Ibrahim, Rosiyah Yahya, Entesar A. Sulliman, Asian journal of chemistry; vol. 24, no. 1 (2012), 269-272.

[16] Ammar A. Ibrahim, Asian journal of chemistry; vol. 23, no. 1 (2011), 215-218.

[17] J. R. Miller and D. E. Edmondson, Biochemistry, 38, 13670 (1999).

[18] Nandigama, R. K.; Edmondson, D. E. Biochemistry 2000, 39, 15258.

[19] Shuai Zhao, Chao Liu, Yong Guo, Ji-Chang Xiao, and Qing-Yun Chen, J. Org. Chem. 2014, 79, 8926-8931

[20] Ye Feng Wang, Jing Hui Zeng, Xiao Rui Cui, Org. Commun. 6:2 (2013) 68-77.

[21] Silvio Cunha, Luis Fernandes P. Santos e Zênis N. Rocha, Roberto Rivelino, Jailton Ferrari, Ivo Vencato e Carlito Lariucci, Quim. Nova, Vol. 33, No. 10, 2108-2112, 2010.

[22] Madhuresh K. Sethi, Somashekar R. Bhandya, Nagaraj Maddur, Sanjay Mahajan, Anish Kumar, Rohit Shukla, Purbita Chakraborty, Der Pharma Chemica, 2016, 8(2):103-108.

[23] Gaoyang Ye, Thermodynamic and structural investigations on the interactions between actinides and phosphonate-based ligands, Ph.D Thesis, Université Paris-Saclay, 2018.

[24] Ondrej Jandourek, Marek Tauchman, Pavla Paterova, Klara Konecna, Lucie Navratilova, Vladimir Kubicek, Ondrej Holas, Jan Zitko, Martin Dolezal, Molecules 2017, 22, 223.

[25] Lucia Semelkova, Ondrej Jand'ourek, Klara Konecna, Pavla Paterova, Lucie Navratilova, Frantisek Trejtnar, Vladimir Kubicek, Jiri Kunes, Martin Dolezal, Jan Zitko, Molecules 2017, 22, 495.

[26] World Health Organisation. Global Action Plan on Antimicrobial Resistance; WHA68/2015/REC/1; World Health Organisation: Geneva, Switzerland, 2015. Available online: http://www.who.int/antimicrobialresistance/publications/global-action-plan/en/ (accessed on 16 January 2017).

[27] Mairi I. McAllister,Cedric Boulho, Lauren F. Gilpin, Liam McMillan, Colin Brennan, David Lennon, Org. Process Res. Dev. 2019, 23, 977-989.

[28] Ivan Juranic, Croat. Chem. Acta 87 (4) (2014) 343-347.

[29] Ilija N. Cvijetic, Maja D. Vitorovic-Todorovic, Ivan O. Juranic, Dura J. Nakarada, Milica D. Milosavljevi, Branko J. Drakulic, Monatsh Chem (2014) 145:1297-1306.

[30] Raghvendra shukla, Pradeep K Sharma, Laszlo Kotai, Kalyan K Banerji, Proc. Indian Acad. Sci. (Chem. Sci.), Vol. 115, No. 2, April 2003, pp 129-134. 